\title{
Exploring Lithium-ion Battery Performance through in situ Characterization
}

\author{
Dean J. Miller ${ }^{1}$, Arnaud Demortiere ${ }^{1}$, Lifen Wang ${ }^{1}$, Jianguo Wen ${ }^{1}$, Jun Lu$^{2}$, Khalil Amine ${ }^{2}$ \\ 1. Electron Microscopy Center - Center for Nanoscale Materials, Argonne National Laboratory. \\ 2. Chemical Sciences and Engineering Division, Argonne National Laboratory.
}

We have developed an approach for in situ and in operando characterization of single Li-ion battery cathode particles through which a single particle can be characterized in detail during electrochemical cycling, providing a better correlation between performance and microstructural evolution.

In this work, we focused on high capacity cathode materials that have a graded composition, with an Nirich core and an Mn-rich periphery. The concept for these materials with a general composition of $\mathrm{LiNi}_{1-\mathrm{x}-\mathrm{y}} \mathrm{Co}_{\mathrm{y}} \mathrm{Mn}_{\mathrm{x}} \mathrm{O}_{2}$ is that the Ni-rich core provides high capacity while the Mn-rich periphery minimizes detrimental interaction with the electrolyte. [1,2] In addition to high capacity, these materials exhibit better long-term performance with less "fade" in capacity over many cycles compared to, for example, $\mathrm{LiNi}_{0.8} \mathrm{Co}_{0.15} \mathrm{Al}_{0.05} \mathrm{O}_{2}$ ("NCA"), which shows much more significant capacity fade. Our in situ single particle studies suggest one of the mechanisms for capacity fade in NCA is particle fracture that occurs during cycling. [3] In this work, we applied our approach to graded cathode materials to see if this was an important factor in their improved performance.

An example of the electrochemical performance of a single $\mathrm{LiNi}_{1-\mathrm{x}-\mathrm{y}} \mathrm{Co}_{\mathrm{y}} \mathrm{Mn}_{\mathrm{x}} \mathrm{O}_{2}$ compositionally graded particle (hereafter referred to a "gradient" material) is shown in Figure 1. In this experiment, the single particle was cycled in situ, and the microstructure was evaluated at various points during cycling. The voltage versus discharge capacity plot shows an increase in capacity for the first 13 cycles, but then a decrease for the 14th cycle. The microstructure of the particle after the 14th cycle, shown in the inset, reveals a moderate degree of cracking (grain-to-grain separations). This microstructure is generally similar to that observed in NCA, but with a notable difference in that the particle cracking tends to be radial rather than isotropic, as is the case for NCA.

This difference is illustrated more definitively in Figure 2, which shows a comparison between gradient material and NCA material for both in situ cycled single particles and for particles harvested from cycled coin cells. This data confirms the aggressive, isotropic fracture mode in NCA that can lead to electrical isolation of individual grains. In contrast, the gradient material exhibits a much more fractureresistant microstructure with anisotropic fracture that should be less deleterious to performance. This comparison also confirms that the behavior we observe in our single particle measurements is indeed representative of larger scale "real world" battery systems. We suggest that the microstructural basis for the radial fracture in gradient materials is based on their grain structure and morphology. The gradient materials exhibit a radial grain structure and refined grain sizes that are more resistant to fracture. Furthermore, the fractures that do eventually appear are less detrimental to performance because they do not lead to isolated fragments that can lose electrical connectivity, as is the case for NCA.

\section{References:}

[1] Y.-K. Sun, et al., Adv. Funct. Mater. 20, 485-491 (2010)

[2] Y.-K. Sun, et al., Nat. Mater. 11, 942-947 (2012).

[3] D.J. Miller, et al., Adv. Energy Mater. 3 (8) 1098-1103 (2013) 
[4] This work was carried out in the Electron Microscopy Center-Center for Nanoscale Materials, which is supported by the U. S. Department of Energy, Office of Science under Contract No. DE-AC02$06 \mathrm{CH} 11357$

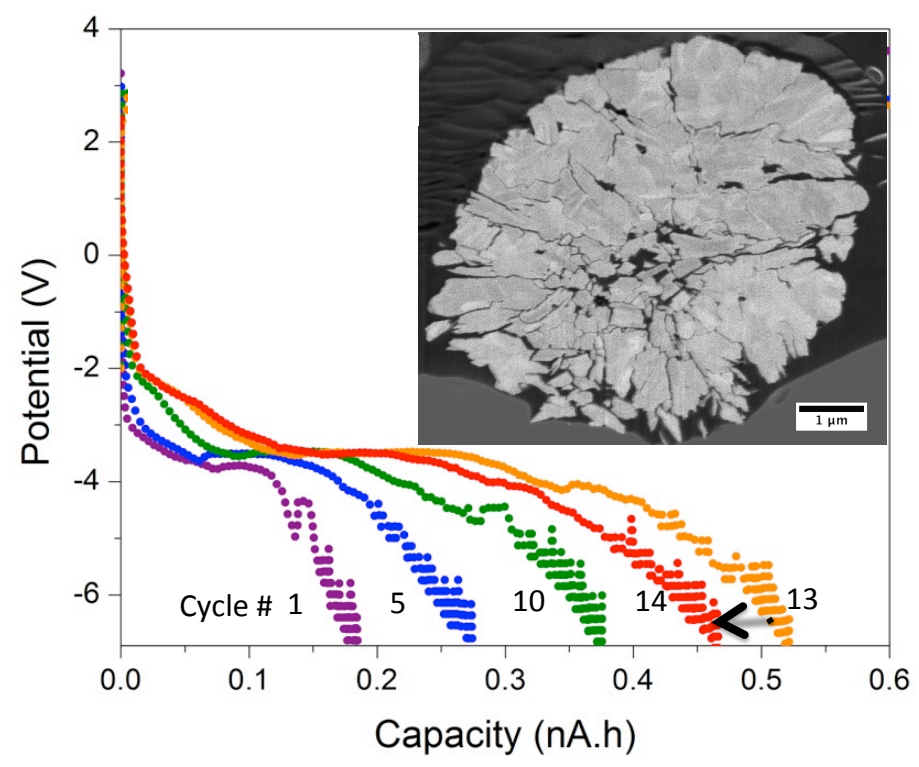

Figure 1. Voltage $v s$ capacity plot measured for a single $\mathrm{LiNi}_{1-\mathrm{x}-\mathrm{y}} \mathrm{Co}_{\mathrm{y}} \mathrm{Mn}_{\mathrm{x}} \mathrm{O}_{2}$ gradient particle and scanning electron microscope image of the particle structure after 14 cycles.

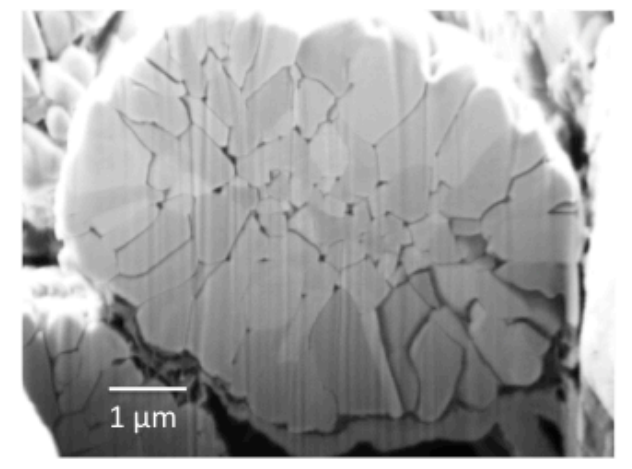

NCA from coin cell - 50 cycles

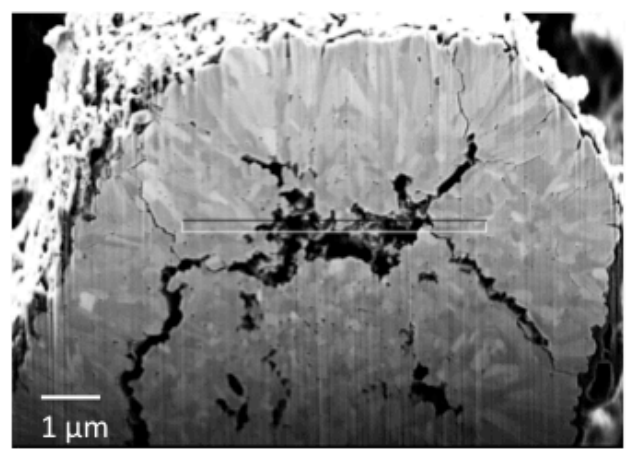

gradient from coin cell - 50 cycles

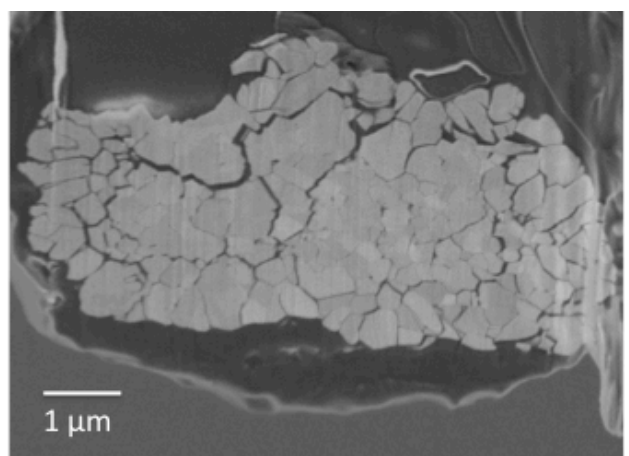

NCA single particle - 3 cycles

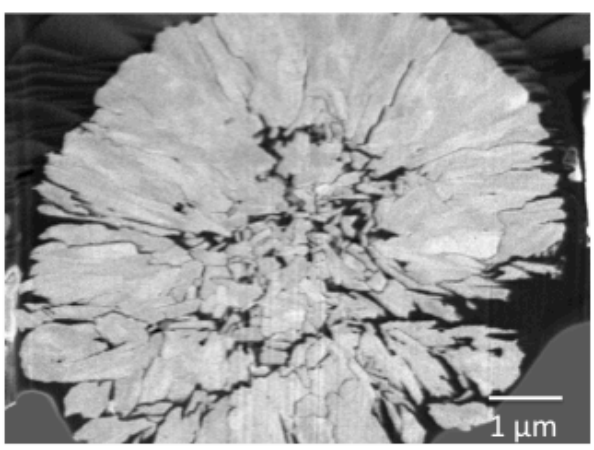

gradient single particle -14 cycles

Figure 2. Scanning electron microscope images of NCA and gradient material after cycling, comparing material cycled 50 times in a coin cell with a corresponding single particle cycling measurement for the number of cycles as indicated. 